\title{
A laser heating facility for energy-dispersive X-ray absorption spectroscopy
}

\author{
Kantor, Innokenty; Marini, C.; Mathon, O.; Pascarelli, S.
}

Published in:

Review of Scientific Instruments

Link to article, DOI:

$10.1063 / 1.5010345$

Publication date:

2018

Document Version

Publisher's PDF, also known as Version of record

Link back to DTU Orbit

Citation (APA):

Kantor, I., Marini, C., Mathon, O., \& Pascarelli, S. (2018). A laser heating facility for energy-dispersive X-ray absorption spectroscopy. Review of Scientific Instruments, 89(1), [013111]. https://doi.org/10.1063/1.5010345

\section{General rights}

Copyright and moral rights for the publications made accessible in the public portal are retained by the authors and/or other copyright owners and it is a condition of accessing publications that users recognise and abide by the legal requirements associated with these rights.

- Users may download and print one copy of any publication from the public portal for the purpose of private study or research.

- You may not further distribute the material or use it for any profit-making activity or commercial gain

- You may freely distribute the URL identifying the publication in the public portal

If you believe that this document breaches copyright please contact us providing details, and we will remove access to the work immediately and investigate your claim. 


\section{A laser heating facility for energy-dispersive X-ray absorption spectroscopy}

I. Kantor, C. Marini, O. Mathon, and S. Pascarelli

Citation: Review of Scientific Instruments 89, 013111 (2018); doi: 10.1063/1.5010345

View online: https://doi.org/10.1063/1.5010345

View Table of Contents: http://aip.scitation.org/toc/rsi/89/1

Published by the American Institute of Physics

\section{Articles you may be interested in}

Soft x-ray spectroscopy of high pressure liquid

Review of Scientific Instruments 89, 013114 (2018); 10.1063/1.5008444

A sample holder for simultaneous Raman and neutron vibrational spectroscopy

Review of Scientific Instruments 89, 013112 (2018); 10.1063/1.4997933

An experimental platform for real-time measurement of the deformation of nuclear fuel rod claddings submitted to thermal transients

Review of Scientific Instruments 89, 013110 (2018); 10.1063/1.4996611

Simultaneous high-pressure high-temperature elastic velocity measurement system up to $27 \mathrm{GPa}$ and 1873 $\mathrm{K}$ using ultrasonic and synchrotron X-ray techniques

Review of Scientific Instruments 89, 014501 (2018); 10.1063/1.4993121

Direct intensity calibration of X-ray grazing-incidence microscopes with home-lab source

Review of Scientific Instruments 89, 013704 (2018); 10.1063/1.5003959

Graphical method for analyzing wide-angle x-ray diffraction

Review of Scientific Instruments 89, 013904 (2018); 10.1063/1.5003452

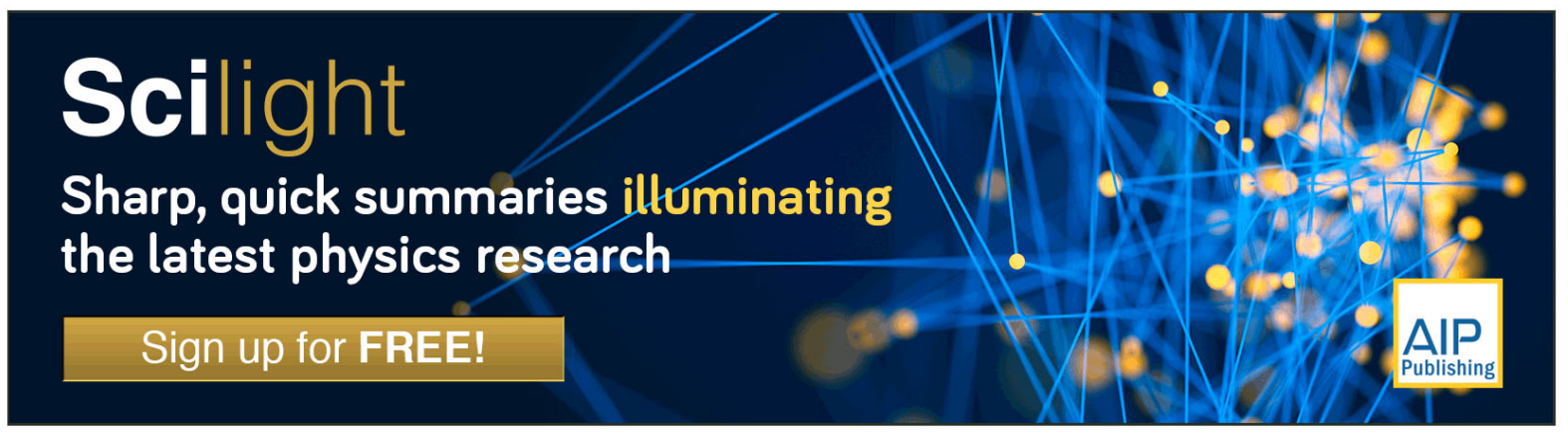




\title{
A laser heating facility for energy-dispersive X-ray absorption spectroscopy
}

\author{
I. Kantor, ${ }^{1,2}$ C. Marini, ${ }^{1,3}$ O. Mathon, ${ }^{1}$ and S. Pascarelli ${ }^{1}$ \\ ${ }^{1}$ ESRF-The European Synchrotron, CS40220, 38043 Grenoble Cedex 9, France \\ ${ }^{2}$ Department of Physics, Technical University of Denmark, Fysikvej 307, 2800 Kongens Lyngby, Denmark \\ ${ }^{3}$ ALBA Synchrotron, Carrer de la Llum 2-26, 08290 Cerdanyola del Vallès, Barcelona, Spain
}

(Received 24 October 2017; accepted 24 December 2017; published online 23 January 2018)

\begin{abstract}
A double-sided laser heating setup for diamond anvil cells installed on the ID24 beamline of the ESRF is presented here. The setup geometry is specially adopted for the needs of energy-dispersive X-ray absorption spectroscopic (XAS) studies of materials under extreme pressure and temperature conditions. We illustrate the performance of the facility with a study on metallic nickel at $60 \mathrm{GPa}$. The XAS data provide the temperature of the melting onset and quantitative information on the structural parameters of the first coordination shell in the hot solid up to melting. Published by AIP Publishing. https://doi.org/10.1063/1.5010345
\end{abstract}

\section{INTRODUCTION}

Today diamond anvil cells (DACs) have become a standard tool for high-pressure studies performed in-house or in large scale facilities. Diamond anvil cells are used routinely at pressures exceeding $1 \mathrm{Mbar}$ in a broad field of scientific applications, ranging from life sciences and chemistry to solid-state physics and material and planetary sciences. A number of experimental techniques have been applied to samples in the DAC, including various types of transmission, absorption, and scattering studies of visible and infrared lights, X-rays, and neutrons. Due to the transparency of diamonds to hard X-rays and the brilliance of modern synchrotron radiation sources, not only X-ray diffraction (XRD), but also different types of X-ray spectroscopies have been applied to samples in the DAC, including X-ray Absorption Spectroscopy (XAS). ${ }^{1}$ Many scientific cases involving high pressures and DAC also require high temperatures for stress annealing, inducing phase transformations and chemical reactions, studying of different physical phenomena, or reproducing P-T conditions of planetary interiors. The easiest and most convenient way to reach temperature exceeding $\sim 1000 \mathrm{~K}$ is to use high power laser heating of the sample inside a DAC.

There are a number of examples of successful coupling of in situ laser-heating systems with synchrotron radiation facilities to probe matter at extreme conditions of pressures and temperatures by means of DACs, and they are extensively used by the community. Some examples are as follows: at the ESRF (beamline ID27), ${ }^{2}$ at the Advanced Photon Source (APS), ${ }^{3-5}$ and at SPring- $8 .{ }^{6}$ However, none are dedicated to $\mathrm{X}$-ray absorption spectroscopy.

In this paper, we describe the first laser heating system for DACs integrated into a XAS synchrotron beamline, providing the users' community a facility for the investigation of the electronic and local structure of matter at very high pressures and temperatures $(\mathrm{P}>100 \mathrm{GPa}, \mathrm{T}>3000 \mathrm{~K})$. This achievement has recently become possible, thanks to a major upgrade of the Energy Dispersive XAS (EDXAS) beamline ID24 of the ESRF. ${ }^{7}$ The in situ investigation of the electronic and local structure of matter at pressures and temperatures relevant to
Earth's interior was in fact chosen as one of the major scientific drivers for the upgrade of this beamline.

The paper is organized as follows. In Sec. II, the XAS and EDXAS techniques are briefly introduced, together with the experimental challenges related to studies of matter at extreme $\mathrm{P}$ and T. In Sec. III, a general technical description of the setup is given. Section IV provides a detailed description of temperature measurement. Section V illustrates the performance of the system through an example on the investigation of melting of $\mathrm{Ni}$, and finally Sec. VI reports conclusions and outlook.

\section{ENERGY DISPERSIVE X-RAY ABSORPTION SPECTROSCOPY APPLIED TO HIGH PRESSURE AND HIGH TEMPERATURE STUDIES}

XAS is a unique tool for probing electronic structure and local atomic environment around a given element in molecules and condensed matter. High-brilliance third generation synchrotron sources have opened new opportunities for XAS studies of condensed matter under extreme conditions of pressure and/or temperature. The first XAS experiment at high pressure using synchrotron radiation took place at Stanford Synchrotron Radiation Laboratory and dates to $1978 .{ }^{8}$ The possibility to provide information on the local structure-through the Extended X-ray Absorption Fine Structure (EXAFS) - and on the electronic structure-through the X-ray Absorption Near Edge Structure (XANES) - makes this technique very complementary to XRD, which is by far the predominant technique for structural studies at high pressure.

The experimental requirements for investigations using the laser heated DAC are stringent. First of all, samples need to be very small $(\sim 10-20 \mu \mathrm{m})$ in order to reach $\mathrm{P}>100 \mathrm{GPa}$. The laser beams need to be focused down to few tens of microns in order to reach the required temperatures. The relative position between the sample, the laser spot, and the X-ray beam must remain stable to within $\sim 1 \mu \mathrm{m}$ to cope with the inevitable pressure and temperature gradients. This leads to the requirement of a very small ( $\sim$ few $\mu \mathrm{m}$ full size) and stable X-ray focal spot. Moreover, the acquisition time needs to be very short, ideally of the order of milliseconds. Molten matter is unstable under in situ laser heating, although much effort is being made to 
reduce this problem. ${ }^{9,10}$ Chemical reactions or decompositions occur at extreme conditions which alter the chemical state and speciation of the absorber. Important temperature and pressure gradients across the laser heated spot are present ${ }^{11}$ and need to be quantified using 2D mapping methods.

These requirements for X-ray spot size, stability, and acquisition speed have hindered up to now the exploitation of XAS as a characterization method for matter at extreme pressures and temperatures because they are very difficult to achieve simultaneously using conventional energy scanning $\mathrm{X}$-ray absorption spectrometers.

In EDXAS, curved crystal energy disperses and focuses a polychromatic X-ray beam onto the sample. The energy bandwidth $\Delta \mathrm{E}$ of the diffracted polychromatic fan of radiation is typically of the order of $0.01 \times \mathrm{E}$, where $\mathrm{E}$ is the central energy. The transmitted beam is then detected by a position sensitive detector, where the energy-direction correlation is transformed into an energy-position correlation. The main advantages of EDXAS spectrometers with respect to conventional energy scanning spectrometers are as follows: 1 -the speed of acquisition, since all energy points are acquired in parallel on the position sensitive detector, and 2-the stability of the energy scale and focal spot position, since there are no moving components during acquisition. Although EDXAS was principally aimed at time-resolved XAS applications, the focusing properties of the polychromator crystal and the absence of movements of the optics made these spectrometers very attractive for other experiments. ${ }^{12}$ The small and stable focal spot was exploited in particular for studies at extreme conditions of pressure using a diamond-anvil cell (DAC). Pioneering work in this field was carried out at beamline D11 at LURE. ${ }^{13,14}$ Further progress in X-ray focusing optics ${ }^{15-17}$ coupled to the high brilliance of the undulator source on ID24 allowed the limit to be pushed up to Mbar and above. ${ }^{18-22}$ First pilot experiments using portable laser-heated DAC systems have been performed to investigate melting of $\mathrm{Fe}$ up to $3000 \mathrm{~K}$ and $80 \mathrm{GPa}^{23}$ and the chemistry of mantle minerals at high pressures and temperatures. ${ }^{24}$ EDXAS is ideally suited to attack these challenging studies because contrary to the energy scanning spectrometers, acquisition speed and stability in the focal spot and energy scale are not linked.

The upgrade provided the opportunity for the development of the first fully dedicated in situ laser heating facility described in Secs. III-VI. This facility is now in full user operation. A first milestone has recently been achieved with the investigation of the melting curve of $\mathrm{Fe}$ at $\mathrm{Mbar}^{25}$

\section{LASER HEATING SYSTEM FOR THE DAC: GENERAL PRINCIPLES AND DESIGN}

A general overview of laser heating setups for diamond anvil cells was recently reported by Salamat and co-authors. ${ }^{26}$ Here we present the general principles of these facilities and discuss in detail the design chosen and implemented on beamline ID24 at the ESRF.

The principal design of a DAC laser heating setup is defined by three main factors: i-laser beam delivery; iisample imaging and temperature measurement optics; and iii-coupling of the setup with the desired experimental technique. The last factor is not important when no in situ sample characterization is performed, e.g., laser heating is used for annealing or phase transformation. In this case, the optical design is significantly simplified. In many cases, however, especially when the system is designed for use in a synchrotron source (e.g., coupled with powder or single-crystal X-ray diffraction, X-ray absorption spectroscopy, and inelastic Xray scattering), the latter factor implies significant constraints on the system design.

Due to progress in performance of solid state diodepumped fiber coupled near-infrared lasers based on Nd-doped YAG (yttrium aluminum garnet), YLF (lithium fluorite), or orthovanadate crystals, these systems have become the most popular radiation source for DAC laser heating systems. They are compact, inexpensive, and convenient for use due to the fiber coupling. They also provide an exceptional quality Gaussian-shaped laser beam with extremely good stability in both the continuous and pulsed mode and the variable output power from fraction of a watt to over $100 \mathrm{~W}$ with a simple air cooling of the main unit. The typical wavelength of these lasers, $1064 \mathrm{~nm}$, allows the use of standard glass optics (with proper antireflection coating). The light of these lasers is efficiently absorbed by most dark or non-transparent samples, while the absorption is negligible by diamond anvils and most common pressure-transmitting and thermal insulating media (noble gases, alkali halides, and simple oxides like $\mathrm{MgO}, \mathrm{SiO}_{2}$, or $\mathrm{Al}_{2} \mathrm{O}_{3}$ ). When sample absorbance in the $1 \mu \mathrm{m}$ wavelength region is low (that is the case for many non-metallic samples), its direct heating can be difficult or impossible due to a low laser coupling. In this case, the sample can be heated indirectly via an inert absorber mixed and compacted with a sample material. Alternatively, long-wavelength infrared lasers, such as $\mathrm{CO}_{2}$ gas lasers with a wavelength of about $10 \mu \mathrm{m}$, can be used at the expense of additional technical difficulties. The most popular choice today, as mentioned above, is the Nd:YAG or similar near-infrared laser. In the ID24 setup, two $120 \mathrm{~W}$ Nd:YAG fiber-coupled lasers manufactured by IPG Photonics have been selected. These lasers can be remotely controlled via a serial line interface; however, a manual external analog control has been found to be more practical. All main laser parameters (switching on and off, starting and stopping the emission, smooth output power regulation, and enabling external modulation) can be modified with knobs and buttons through a small control box.

The laser beam delivery is the simplest but an important part of the system. The laser needs to be focused on the sample and needs to be position-adjustable in order to heat the right portion of the sample. The laser beam can be delivered either via independent optics or by sharing a common objective with the imaging part of the setup. In the first case, the setup is much more flexible: the laser beam position can be adjusted independently from the imaging part of the optics, and the laser spot size can be varied by simply defocusing the laser beam. The latter case, however, is more user-friendly because laser beams are initially pre-aligned with the sample image, and users do not need to check the laser beam position relative to the image before heating. An alternative solution to the problem of generating relatively large (15-20 $\mu \mathrm{m})$ uniform and homogeneous 
laser spots is the use of special focal beam-profiling optics (П-shapers $)^{10}$ which allows reaching similar uniform power density in a central spot for a lower total laser power. On the ID24 system, IR lasers are focused on the sample using two separate simple plano-convex lenses with $0.5 \mathrm{in}$. diameter and a focal distance of $50 \mathrm{~mm}$ (Fig. 1). For the laser spot position adjustment, the lens can be translated in three directions. The horizontal and vertical translations are performed with the manually controlled DC motors, while focusing is done with a miniature stepper motor for precise tracking of the sample focal position.

Sample imaging coupled with temperature measurements is the most critical part of the setup. Samples in diamond anvil cell experiments are typically less than $0.1 \mathrm{~mm}$ in size and often are as small as a few micrometers. Therefore, the laser heating system has to provide a high quality, sharp, highly magnified image to enable a precise alignment. Temperature measurements are of great importance in DAC laser heating experiments, and today, systematic errors in temperature measurements are probably responsible for many discrepancies between different experimental studies. The most common way to measure temperature in situ is the optical micropyrometry (or imaging spectroradiometry). The thermal emission spectrum from the sample is collected with a microscope objective and analyzed using Planck's law, in most cases with the grey-body approximation. Planck's law describes electromagnetic radiation emitted by a black body in thermal equilibrium at any finite temperature as a function of wavelength $\lambda$ and temperature $T$,

$$
B=\frac{2 h c^{2}}{\lambda^{5}\left(e^{\frac{h c}{\lambda k_{B} T}}-1\right)},
$$

where $B$ is the absolute spectral radiance, and $h, c$, and $k_{B}$ are the Planck constant, the speed of light, and the Boltzmann constant correspondingly. In real substances, in most cases, the observed spectral radiance is smaller, so an empirical emittance coefficient $\varepsilon$ is introduced $(\varepsilon \leq 1)$. When emittance is wavelength independent, such a material is called a grey body. Planck's function has an asymmetric bell-like
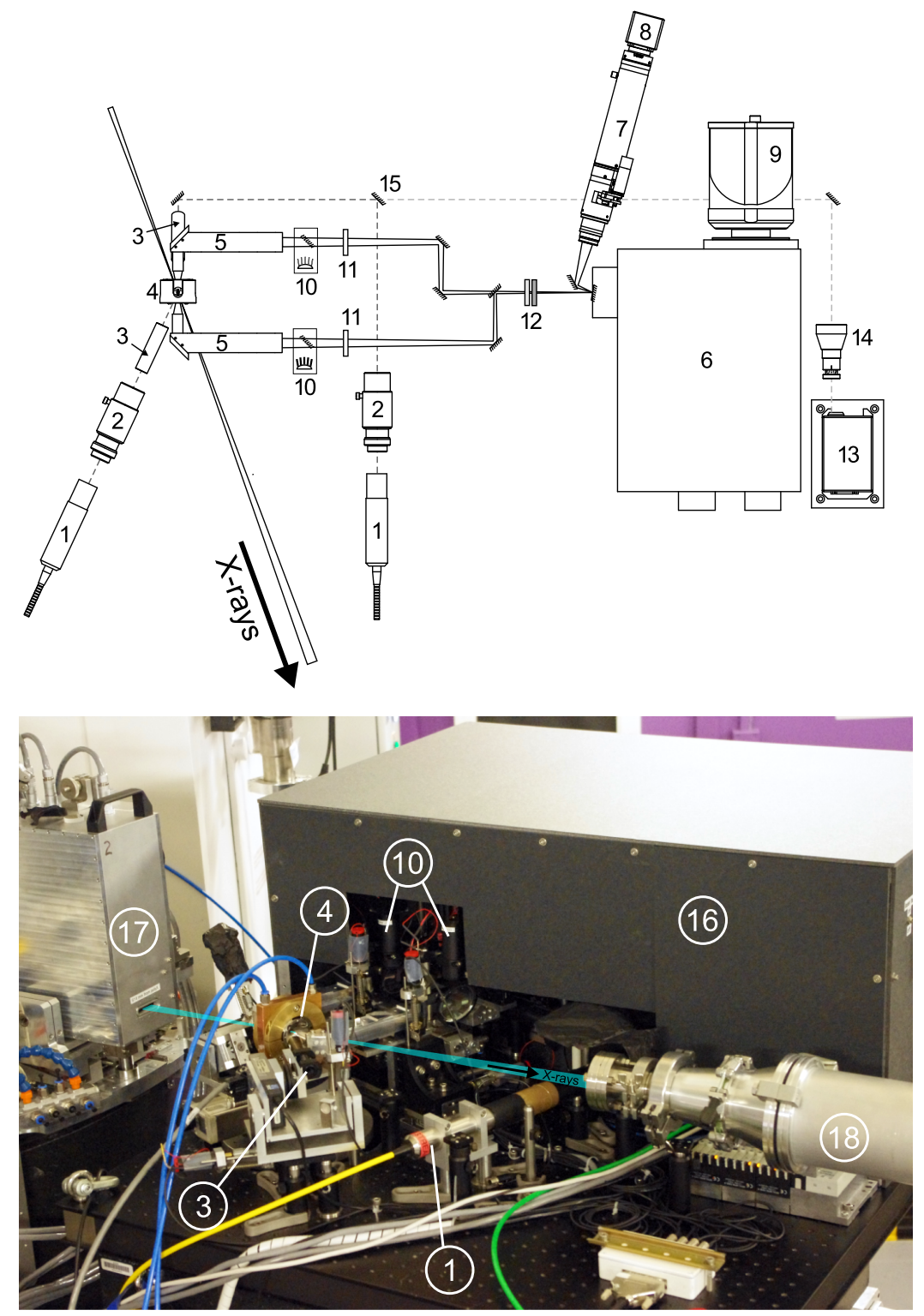

FIG. 1. Schematic layout and a photograph of the laser heating setup for high pressure and high temperature $\mathrm{X}$-ray absorption spectroscopy at beamline ID24 at the ESRF. (1)—IR fiber lasers heads; (2) — beam-profilers for flat-top heating (optional); (3)-laser focusing lens $(f=50 \mathrm{~mm}) ;(4)$-diamond anvil cell; (5)—sample imaging objective; (6) - Czerny-Turner type optical spectrometer $(f=300 \mathrm{~mm}) ;(7)-6 \mathrm{X}$ motorized zoom microscope; (8)—digital color video CCD camera; (9)spectrometer camera; (10)_LED illumination modules; (11) - removable laser notch filters for 532 and $1062 \mathrm{~nm}$; (12)—neutral density grey optical filters; (13) - green $532 \mathrm{~nm}$ laser for Raman and fluorescence excitation; (14)-beam expander for the green laser; (15) - removable laser mirror to switch between heating and fluorescence measurements; (16)—optical enclosure for stray light and dust protection; (17) - X-ray vertical focusing mirror; (18) - vacuum tube connected to the $\mathrm{X}$ ray detector, which is at approximately $2 \mathrm{~m}$ distance from the sample. 
shape with a steeper low-wavelength part. For typical temperatures of a DAC laser-heating experiment $(2000-5000 \mathrm{~K})$, the most appropriate radiometry wavelength range is visible to the near-infrared region.

For the short wavelength limit when $h c / \lambda \gg k_{B} T$, Eq. (1) can be re-written as

$$
B=\frac{2 h c^{2}}{\lambda^{5}\left(e^{\frac{h c}{\lambda k_{B} T}}\right)},
$$

known as the Wien approximation. For typical DAC laser heating experimental conditions $[400<\lambda(\mathrm{nm})<1000, \mathrm{~T}<$ $5000 \mathrm{~K}$ ], the difference between the Planck and Wien functions is very small. For example, in the case of a grey-body emission for a 400-1000 nm fitting range, the Wien fit would give a temperature offset of $\sim-3 \mathrm{~K}$ for $\mathrm{T}=3000 \mathrm{~K}$ and $\sim-64 \mathrm{~K}$ at $\mathrm{T}=5000 \mathrm{~K}$. Temperature fitting errors of $\sim 1 \%$ between the Planck and Wien fits are negligible compared to other sources of uncertainties. Walter and $\mathrm{Koga}^{27}$ claimed that the Wien function could be more precise when emissivity linearly varies with the wavelength. However, that is not necessarily true, and depending on the emissivity slope, temperature, and fitting range, it can give either slightly higher or lower accuracy compared to the Planck fit. For example, for $\mathrm{T}=3000 \mathrm{~K}$ and for the same 400-1000 nm fitting range, assuming a $20 \%$ linear decrease in emissivity across the fitting range, the Wien fit gives temperatures $40 \mathrm{~K}$ closer to the real one compared to the Planck fit. At the same time, at $\mathrm{T}=5000 \mathrm{~K}$ and assuming a $10 \%$ linear increase in emissivity, the situation is inversed, and the Planck fit provides temperature values that are $61 \mathrm{~K}$ closer in comparison to the Wien fit. Therefore, we recommend always using the Planck function (1) for temperature fits, especially for temperatures above $\sim 3000 \mathrm{~K}$, when the Wien approximation becomes less accurate. The potential effect of the wavelength dependence of emissivity on a temperature measurement is discussed briefly further below.

To create a magnified image of the sample, a microscope is required. Most often a double-lens (refractive) microscope is used for this purpose because it is simple and compact, has affordable price, and can produce an excellent image of sufficiently high magnification. A reflective (Schwarzschild design) objective has been also proposed ${ }^{2}$ as an alternative due to its intrinsic pure achromatic properties. Nevertheless, the ID24 system has been equipped with refractive lenses, and the justification for this choice is given below.

Sample imaging is performed in two stages, following the principal scheme suggested by Boehler and co-authors. ${ }^{23}$ The primary imaging stage projects the real sample image on the optical spectrometer entrance slit using a pair of achromatic lenses, mounted in a custom-made right-angle objective (5 in Fig. 1). The entrance slit of the spectrometer is replaced with a metallic mirror with two small $(\sim 35 \mu \mathrm{m})$ holes. The holes are separated vertically by $\sim 4 \mathrm{~mm}$ and are used to collect the light from both sides of the sample into the spectrometer simultaneously. By selecting two independent regions of interest on a two-dimensional charge-coupled device (CCD) spectrometer camera, temperatures on both sides of the sample are collected simultaneously in a single exposure. On the secondary imaging stage, the light, reflected by the spectrometer entrance mirror is then directed in the microscope ( 7 in Fig. 1), equipped with a high-sensitivity color digital video camera (8 in Fig. 1). By tilting the spectrometer entrance mirror by $\sim 5^{\circ}$, the reflected light is deviated from the principal optical axis, thus avoiding using any additional beam splitters. Such geometry increases the system throughput and minimizes any possible distortions or parasitic light reflections from additional interface surfaces. The secondary imaging microscope (produced by Navitar) is equipped with a motorized 6-fold optical zoom module for alignment purposes. This imaging microscope is mounted on a vertical precision translation stage driven by a fast pneumatic actuator, which allows users to switch between images of two sides of a DAC within a second. The infrared cutoff filter, mounted in front of the sensor of virtually any commercially available camera, is removed and replaced with a movable filter to allow IR laser alignment at low power, as described below.

For the spectral analysis, a Czerny-Turner type imaging spectrometer with a $300 \mathrm{~mm}$ focal length is utilized, coupled with a $1340 \times 400$ pixel Pixis back-illuminated CCD camera, both produced by Princeton Instruments. An important remark concerning the location of the mechanical shutter should be made: the shutter should be mounted just after the entrance hole(s) of the spectrometer and not in front of the CCD camera. This avoids the "diaphragm" effect of relative intensity loss at the spectral margins on short exposures due to the iris-like shutter design. The spectrometer turret carries three different diffraction gratings, so the same spectrometer and optical path can be used for temperature measurements (when a wide wavelength range is desired) and pressure determination using ruby fluorescence or diamond anvil Raman signals (when a high spectral resolution is necessary). The rotating spectrometer turret allows switching between the temperature measuring mode and spectroscopy mode in few seconds. For the fluorescence and/or Raman excitation, we use a solid-state Raman-grade 532-nm laser (13 in Fig. 1), although ruby fluorescence can also be successfully excited with the intense X-ray beam even at a megabar pressure.

In an optical scheme with two achromat lenses, it is essential to keep perfect relative alignment (coaxiality and parallelism) between both lenses. In order to keep the lenses fixed during adjustments of the optics, a small objective unit holds the two lenses and a front-surface mirror that creates a right angle between them (Fig. 2).

Illumination is also an important part of the imaging system. A collimated ultra-bright white LED (light-emitting diode) is utilized as a cheap and efficient light source, which dissipates a very small amount of heat and does not require active cooling. Thin-film "pellicle" beamsplitters send the light into the objectives. Such beamsplitters are ideal in this application because they have a virtually zero thickness, thus introducing no light displacement or deviation. The whole illumination unit moves in and out of the optical beam for sample observation and alignment and for $T$ measurements.

When the sample temperature is high (above $\sim 3000 \mathrm{~K}$ ), the spectrometer CCD will be saturated even at the shortest exposure time. To reduce the light intensity, we use two grey 


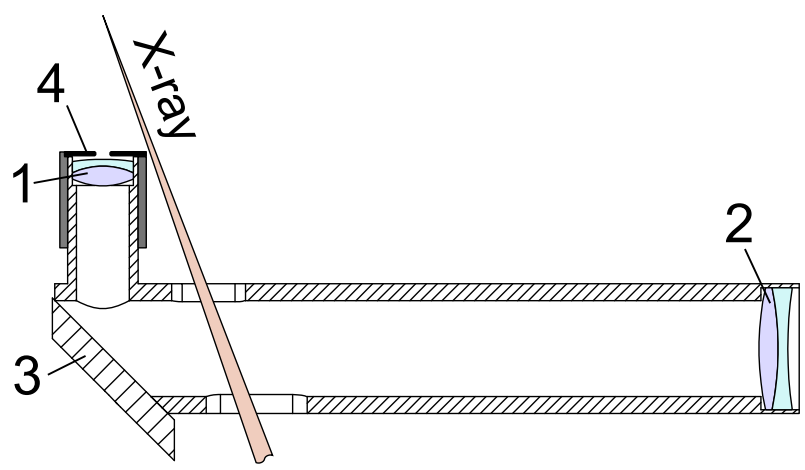

FIG. 2. Custom-built objective unit that holds the front achromatic lens with $30 \mathrm{~mm}$ focal distance (1) and a back achromatic lens with $500 \mathrm{~mm}$ focal distance (3), along with a front surface mirror (3) and a removable aperture (4). Note the two openings in the objective body, which allow the X-ray beam to pass through.

neutral density glass filters with optical densities of 1 and 2 , allowing varying light transmittance between $100 \%, 10 \%, 1 \%$, and $0.1 \%$.

One of the most critical issues of a laser heating experiment concerns the relative alignment between the X-ray, the laser beam, and the temperature-probed sample area. The alignment procedure consists of three consecutive steps as described below.
1. Since EDXAS is very sensitive to sample quality and thickness homogeneity, the first step in the alignment procedure consists of finding a spot on the sample that gives good quality XAS spectra. The first direction of optimization is along the X-ray beam propagation because the sample must be placed on the focal plane of the highly diverging polychromatic beam. This is done by performing scans of the cell in the horizontal direction perpendicular to the beam, using the borders of the gasket as "knife edges," until the sharpest profile is measured on the transmitted beam. The focal depth of the polychromatic fan of radiation is of the order of $100 \mu \mathrm{m}$; therefore the " $x$ " coordinate is optimized with this precision. We then scan along the other two coordinates $(y, z)$ and search for the most homogeneous region of the sample. Once this spot is identified, the sample position relative to the X-ray intersection position on the sample (also referred to as "X-ray spot on the sample" or more simply "X-ray spot") is fixed. The y and z coordinates are optimized with the precision and reproducibility of $\sim 1 \mu \mathrm{m}$.

2. The second step involves tweaking the position of the objective so that the X-ray spot overlaps with the spectrometer entrance hole. This guarantees that the temperature is measured from the exact same spot as the X-ray absorption spectrum.
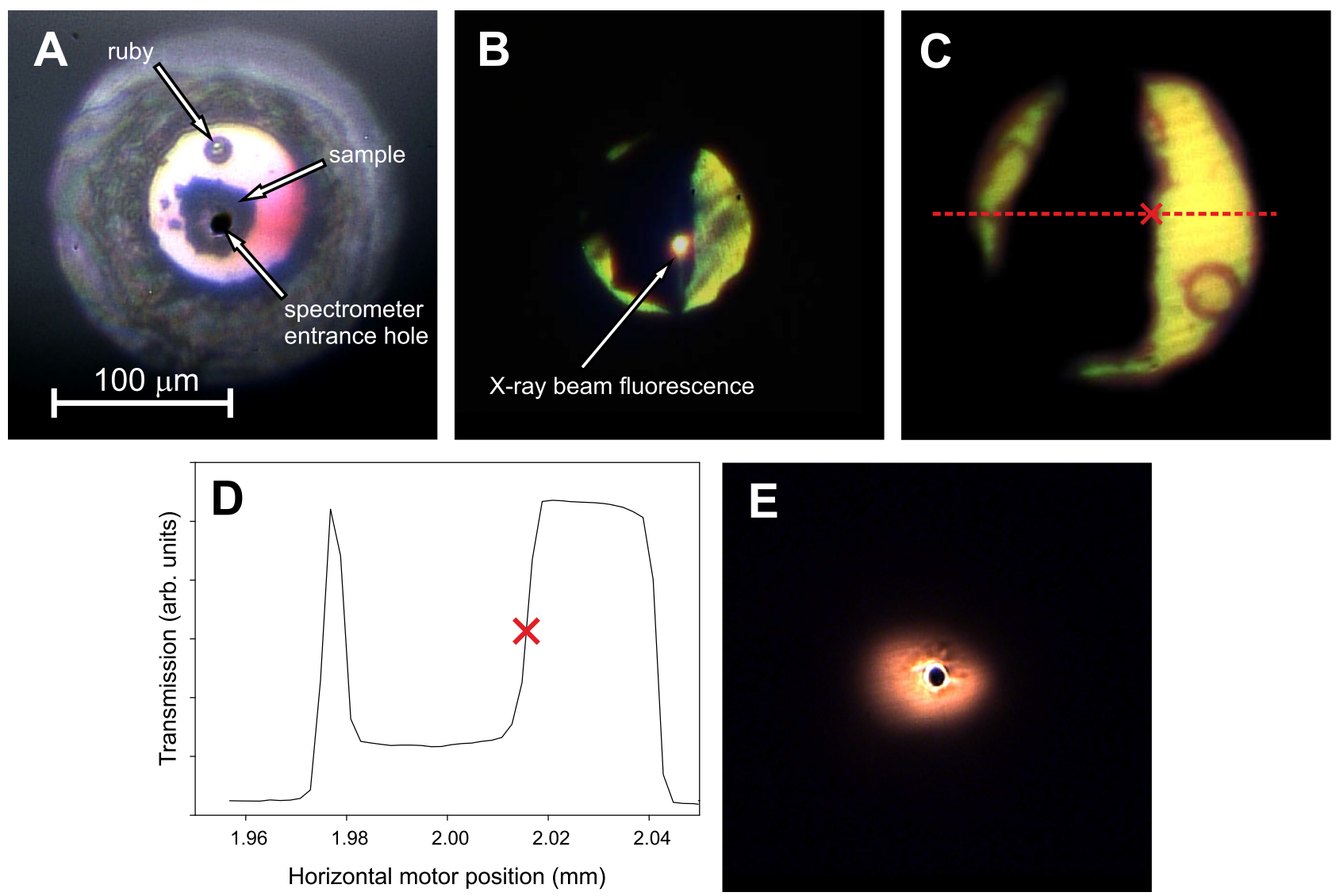

FIG. 3. Diamond anvil cell sample images. (a) Image in both the transmitted light and reflected light. The sample, the ruby sphere, and the spectrometer entrance hole are visible. (b) Fluorescence spot excited with the X-ray beam. (c) Sample image with the dashed red line showing the beam path during the horizontal scan with the corresponding measured X-ray transmission profile shown in (d). The red cross shows the position at the sample edge used to match the visible image with the X-ray beam. (e) Image of the hot spot at $\sim 2500 \mathrm{~K}$. 
The spectrometer entrance hole is visible on the sample image in the form of a small ( 3-4 $\mu \mathrm{m})$ black circle superimposed on the sample image [Fig. 3(a)], as a result of the double-stage optical scheme described above. Thus it is always absolutely clear from what part of the sample the signal is analyzed at any time. The exact localization of the X-ray spot on the sample image requires special alignment. The approximate position of the X-ray beam on the sample is often betrayed by X-ray excited fluorescence along the X-ray path, from the pressuretransmitting medium, the sample, and/or the diamond anvils [Fig. 3(b)]. This fluorescence signal is visible on the upstream and downstream images of the sample with proper camera exposure settings. However, since the Xray beam enters the DAC at an angle of $20^{\circ}$, special attention is needed to avoid parallax errors. This is achieved by performing a horizontal scan of the sample across the Xray beam [Figs. 3(c) and 3(d)]. By placing the horizontal motor position on one of the two sample edge positions [red cross in Fig. 3(d)], the X-ray spot in the horizontal direction is identified with the precision of $\sim 1 \mu \mathrm{m}$ [red cross in Fig. 3(c)]. At this point, the objective can be tweaked until the X-ray spot overlaps with the spectrometer entrance hole. In practice, we have measured that the position of the $\mathrm{X}$-ray beam determined by the scan always coincides with the position of the X-ray beam revealed by the fluorescence. In the vertical direction, there is no parallax effect, and the visible X-ray excited fluorescence spot always coincides with the actual $\mathrm{X}$-ray beam position.

3. The third step is devoted to the alignment of the laser beam. After removal of all the filters (including notch and IR cut-off camera filters), the IR laser becomes clearly visible even at the lowest power (below $100 \mathrm{~mW}$ ), from both the upstream and downstream images of the cell. After inserting the filters and increasing laser power, a hot spot can be clearly seen on the camera due to the thermal emission. The desired size of the hot spot (in most cases, the larger the better) is achieved by defocusing the laser beam (moving the laser focusing lens towards the sample), adjusting at the same time the laser power [Fig. 3(e)]. By small movements of the laser focusing lens perpendicular to its optical axis, the laser spot position on the sample is adjusted, so that the hot spot is centered on the spectrometer entrance pinhole, on both the upstream and the downstream images. All of the movements and tweaks of the diamond anvil cell and optical components are fully motorized, and the alignment procedure is performed remotely from the beamline control cabin.

\section{TEMPERATURE MEASUREMENT: CHROMATIC ABERRATIONS AND SPATIAL RESOLUTION}

Imaging optics can introduce a whole range of geometric, wavefront, and spectral distortions. Typically the temperature is analyzed from a small spot at the center of the image, and in this case, most of these distortions become negligible for temperature measurement purposes, except for chromatic aberrations.
Chromatic aberrations appear due to light dispersion in refractive optics. Refraction index variations as a function of wavelength result in variations of the exact focal distance of a lens for different wavelengths. A specially designed combination of two or more lenses (called achromatic or apochromatic lenses) made of materials with different refraction indexes allows reducing this aberration dramatically for a specific wavelength range. Chromatic aberrations are usually described by focal shift curves, showing the variation of the lens focal distance with wavelength. However, these curves are not very informative for the purposes described here. On one hand, they should be always compared to the focal depth value (as long as the focal shift remains within the focal depth, it is not manifested in the image produced). On the other hand, during the measurements, the sample or the lens is not moved along their axis, so lens spectral properties must be characterized for a fixed distance to the sample. When the image is perfectly infocus for one wavelength, it will be slightly out of focus for another wavelength. This results in an image blurring which can be fairly well described as a 2D Gaussian blurring for circular lens aperture, where a Gaussian width is given by a system point-spread function. This blurring reflects a loss of spatial resolution of the image. Therefore, chromatic aberrations for a fixed focal distance are equal to the optical (spatial) resolution variations as a function of wavelength. Linear optical (or spatial) resolution describes the ability of the imaging system to resolve small objects. The resolution $\delta l$ is usually expressed as the minimal distance at which two bright objects can be resolved. According to the Rayleigh criterion, objects are resolved when the contrast between the maximum intensity and the minimum intensity is larger than $26 \%$. An optical resolution of $\delta l$ means that the projected image (which is a twodimensional intensity distribution over the focal plane) would be convoluted with a Gaussian function with the full width at half maximum of $0.833 \times \delta l .{ }^{28}$ Wavelength dependent variations of the linear resolution would affect temperature fitting. For a sample with an absolutely uniform temperature distribution, the intensity probed at the very center of the final image would be correct as long as the $\delta l$ value is lower than the sample size. At the same time, when the resolution exceeds the sample size, the intensity measured could be significantly (tens of percent) lower than the real one. If the optical linear resolution varies significantly along the fitting wavelength range, and is comparable or larger than the hot spot size, the relative intensities measured and thus the calculated temperature will be incorrect.

The effect of chromatic aberration on temperature measurements depends not only on the choice of optics and wavelength range used, but also on the size and shape (temperature distribution in space) of the analyzed hot spot and the size of the spectrometer entrance hole or the optical fiber core size used to collect the signal. Indeed, when the studied sample has a uniform temperature distribution over an area significantly larger than the system's worst spatial resolution and the spectrometer entrance hole, chromatic aberrations will not have any effect on temperature fitting. This is the case for most light sources used for optical system calibration, such as tungsten filament bulbs, heated thermocouple junctions, or classical black-body oven entrance pinholes. All 
these objects typically have a homogeneous temperature over the area of hundreds of microns or more, while the system spatial resolution is typically ranging from few to tens of microns. Such large etalon objects are perfect for calibrating the instrumental response function, but they cannot be used to analyze the system's chromatic aberrations. Chromatic aberration problems appear only on relatively small and non-uniform hot spots, such as a laser-heated sample in a diamond anvil cell. In order to understand the effect, one needs to characterize the spatial resolution curve of the system. A variation of linear resolution with wavelength is caused, however, not only by chromatic aberrations of refractive optics.

Minimal linear resolution of any optical system is also limited by the diffraction of light. This Rayleigh limit depends on the wavelength and the numerical aperture of the system. The resolution limit of a lens is defined as $\delta l=0.61 \lambda / \mathrm{N}$.A., where $\lambda$ is the wavelength and N.A. is the lens numerical aperture. It is important to notice that due to this fundamental limitation no optical system is truly achromatic, even when only reflective optics is used, since there is in any case a twofold variation of the spatial resolution when comparing blue $(\sim 450 \mathrm{~nm})$ and near-infrared $(\sim 900 \mathrm{~nm})$ parts of the spectrum for a diffraction-limited optical system.

The linear spatial resolution of the system can be calculated using standard ray-tracing software if all optical components are known. However, it is preferable to measure it experimentally in order to include all possible contributions and imperfection of optical elements. A simple method is proposed that allows a fast characterization of the system's achromaticity. A sharp edge is placed on the sample position in the focus of the system, back-illuminated by a continuous light source (such as a filament bulb). A scan of the sharp edge across the image provides intensity variations from which the linear resolution of the system can be derived. Ideally, the light source should also be in the focal plane of the objective-this condition is achieved when scanning the edge of a large $(0.4 \mathrm{~mm}$ or more) core of an optical fiber-and the fiber termination serves as a sharp edge. We performed such scans with a step of $1 \mu \mathrm{m}$. At each step, the intensity as a function of wavelength is recorded by the spectrometer. Figure 4(a) shows the intensity profile as a function of edge position, at different wavelengths within the spectrometer range. A derivative of such a function represents the effective linear resolution of the system for a given wavelength, fitted to a Gaussian function [bottom panel of Fig. 4(a)]. The resulting linear resolution of the system at different wavelengths is shown in Fig. 4(b). As can be seen, the measured resolution curves are fairly close to the theoretical diffraction limit for the corresponding numerical aperture within the wavelength specified by the manufacturer (650-1000 nm) as an achromatic range. At the same time, by reducing the numerical aperture with the front pupil as is suggested by Boehler et al., ${ }^{23}$ the useful range for temperature fitting can be extended down to $\sim 550 \mathrm{~nm}$ at the price of overall resolution loss. The fact that for some wavelengths (e.g., 500 and $550 \mathrm{~nm}$ ) linear resolution is better for smaller aperture seems to be a paradox because as is mentioned above, the optical resolution is in inverse ratio to the numerical aperture. However, this is not necessarily the case for objects that are out of focus: reducing numerical aperture increases the focal depth and such objects became sharper. This effect is well known by the photographers: by reducing the numerical aperture of the objective lens, out-of-focus objects become sharper. The effect of focal depth change can be clearly demonstrated by repeating the same measurements after a small $(20 \mu \mathrm{m})$ translation of the objective away from the real focal plane. As can be seen from the orange and green curves in Fig. 4(b), such small misalignment can produce huge chromatic aberrations when a full numerical aperture is used, while having no or little effect when the aperture is reduced.

It is found that it is often useful to reduce the numerical aperture from 0.2 to $\sim 0.08$ at the price of loss in resolution, not only to increase the useful wavelength range for temperature
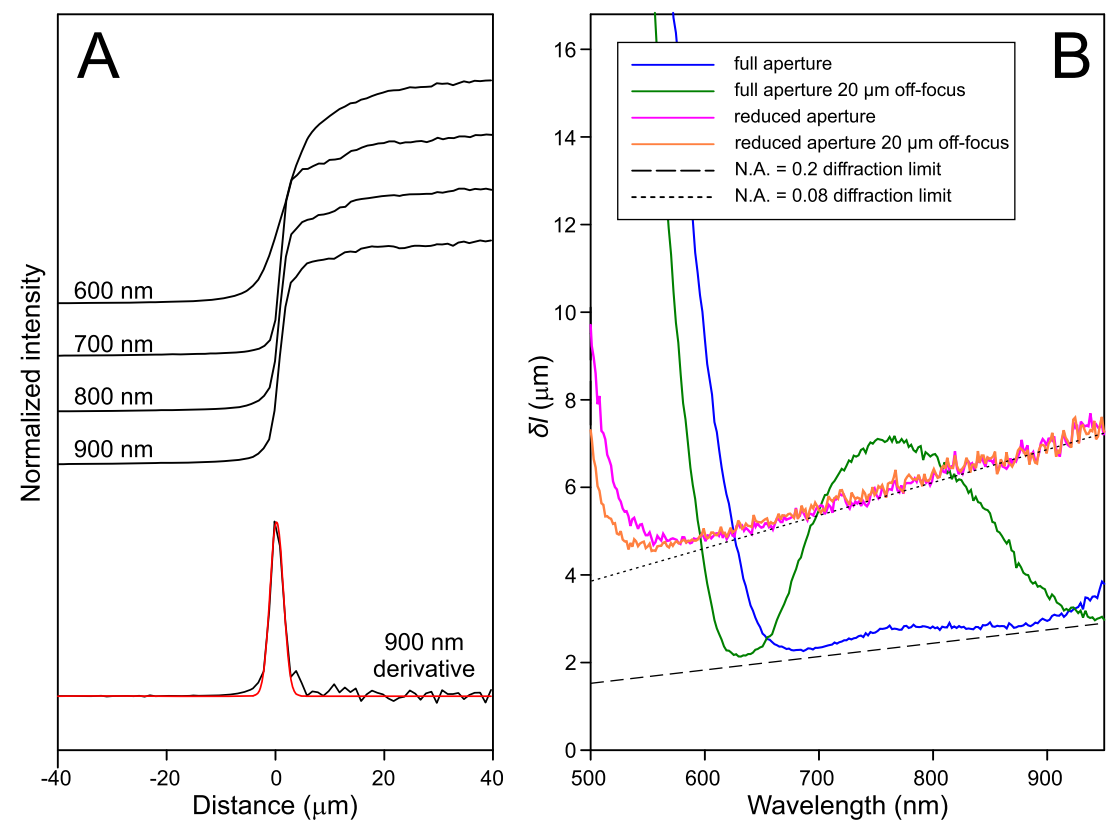

FIG. 4. (a) Sharp edge scans on the optical spectrometer for different wavelengths, using the full front lens aperture. At the bottom, a derivative of one scan $(\lambda=900 \mathrm{~nm})$ and a corresponding Gaussian fit. (b) Extracted resolution curves for full and reduced apertures (blue and pink, respectively) and the corresponding diffraction limit curves for N.A. of 0.2 and 0.08 (long and short-dashed lines, correspondingly). The effect of $20 \mu \mathrm{m}$ focal misalignment is shown with the green and orange curves, respectively. 
fitting but also to decrease fluorescence excited by X-rays and laser beam from the sample, pressure medium, or diamond anvil.

Measuring the wavelength-dependent resolution curves of the system gives a fast way to understand not only the useful wavelength range for temperature fitting but also the minimal size of the uniform hot spot that should be larger than the linear resolution. For experiments at very high pressures (over 100-200 GPa), the sample is generally quite small, and the uniform hot spot dimension is even smaller, of the order of few microns. This limits the accuracy of temperature measurements, and from our estimations, the error introduced by the resolution variation can be as much as several hundred degrees for small or non-uniformly heated samples, depending on the exact temperature profile, system numerical aperture, and wavelength range used.

On the contrary, if the uniform part of the heated sample is large enough ( $\sim 10-15 \mu \mathrm{m}$ or more), a reliable temperature fit can be obtained for a wide range of temperatures (Fig. 5). The lowest temperature that can be measured with the ID24 system is below $1000 \mathrm{~K}$, but this measurement requires relatively long (5-10 $\mathrm{min})$ exposure times due to very low number of photons.

In a real experiment with a diamond anvil cell, the diamond anvil itself is an additional dispersive optical element. At low pressure, the diamond anvil is a flat optical window, thus introducing only minimal chromatic aberrations, despite its high refraction index. At high pressures, the culet of the diamond anvil is known to deform, ${ }^{29,30}$ forming a kind of plano-concave lens. Fortunately, light dispersion inside the diamond anvil is small, and this effect most likely does not introduce significant spectral distortions during temperature measurements, as is confirmed by our preliminary ray-tracing simulations. ${ }^{31}$

To conclude, the key to accurate pyrometric temperature measurements is a large and uniformly heated spot. Several

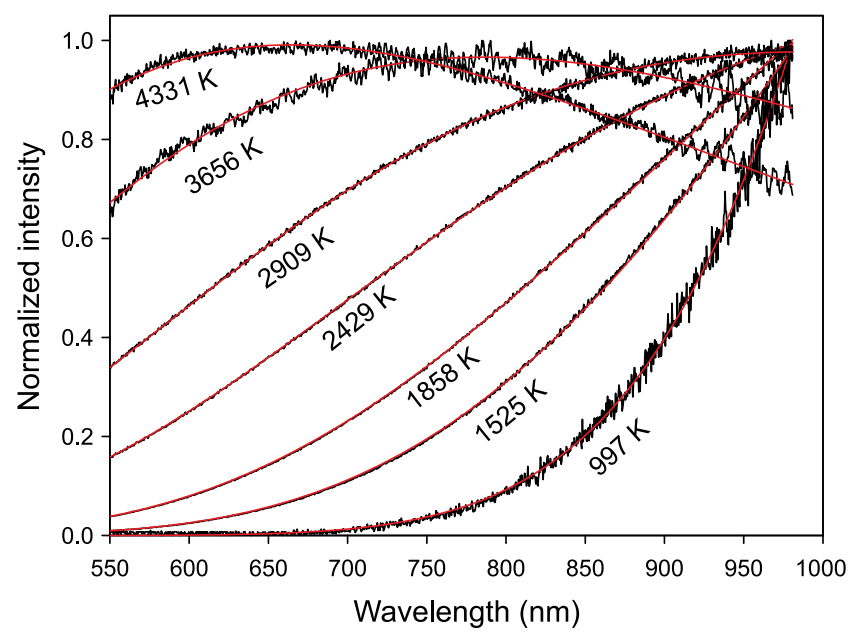

FIG. 5. Examples of the corrected measured emission spectra (black) and corresponding Planck's fits (red) for different temperatures. T values from best fits are shown next to each curve. All intensities are corrected for the instrumental response function and normalized to unity. The lowest temperature has been measured from the tungsten filament bulb, while the two highest curves have been recorded during laser heating of carbon fiber sheets at ambient pressure. studies have been performed to investigate the effect of the sample geometry on lateral and axial temperature gradients in a laser-heated diamond anvil cell. ${ }^{11,32}$ The ideal sample is as thin as possible (preferably less than $10 \mu \mathrm{m}$ ) to minimize axial thermal gradients, with a thick layer of thermal insulation on both sides and relatively large radial dimensions to minimize radial thermal gradients when combined with a large laser spot.

Another source of potential uncertainty of imaging pyrometry is the wavelength dependence of sample spectral emissivity. The value of absolute emissivity depends not only on the material, its surface properties (for example, polishing a metal surface can reduce emissivity by a factor of 5), and temperature but also on the wavelength. Wavelength dependence of emissivity is of fundamental interest for solid state physics but difficult to measure. Most measurements of spectral emissivity are performed in the infrared region $(\lambda>1 \mu \mathrm{m})$; however, some data are available also for the visible and near-infrared region. In the 500-1000 $\mathrm{nm}$ range, emissivity variations (or deviations from the grey-body approximation) usually do not exceed $10 \%$; thus temperature uncertainties are typically expected to be lower than $\sim 100 \mathrm{~K}$, a generally acceptable temperature uncertainty. Some experimentalists introduce linear emissivity variation as a parameter in the temperature fitting routine. This improves fitting quality; however, this assumption cannot be justified because emissivity in a laserheated cell cannot be determined from the emission spectrum independent of temperature values.

\section{APPLICATION EXAMPLE: MELTING OF Ni METAL AT HIGH PRESSURES}

Nickel is believed to be the second most abundant element of the Earth's core after iron, and thus its high-pressure behavior is of great interest for geosciences. Unlike iron, nickel does not undergo any phase transition in the pressure range relevant to the Earth's interior and remains in a magnetically ordered face-centered cubic structure. ${ }^{33,34}$ In Earth sciences, among major physical properties of the interior of our planet, the temperature profile remains one of the least certain, in contrast to pressure or density profiles. One of the most important constraints for the deep Earth geotherm (depth variation of temperature) is the boundary between the inner solid and outer liquid cores, where the temperature matches the melting temperature of the iron-nickel alloy (with some light elements). From this perspective, the high pressure melting curve of nickel is as important as that of iron, and it has been the subject of several experimental studies in recent years. ${ }^{35-38}$

The detection of melting in a laser-heated diamond anvil cell is not a trivial task, and many different criteria have been proposed by experimentalists. Some of them are based on indirect evidence (like temperature variation as a function of laser power), and some use optical properties of the sample surface (laser speckle technique or emissivity discontinuities). Recently, methods based on the direct probe of bulk properties have become accessible. The most common method exploits $\mathrm{X}$-ray diffraction, where melting is detected by the appearance of the diffusion halo from the liquid. Other methods, 
such as nuclear resonance scattering, have also been recently tested. ${ }^{39} \mathrm{X}$-ray absorption spectroscopy, as previously mentioned, is a complementary new powerful tool to study melting phenomena under extreme pressures.

A pure nickel foil (99.9\%) of $5 \mu \mathrm{m}$ thickness from Goodfellow was used as a sample. A piece of $\sim 50 \mu \mathrm{m}$ in diameter was loaded inside a $120 \mu \mathrm{m}$ diameter hole inside a rhenium gasket. Single crystal diamond anvils with $300 \mu \mathrm{m}$ culet size were used, and potassium chloride $\mathrm{KCl}$ was loaded as a pressure transmitting medium and a thermal insulator for the sample. The thickness of the $\mathrm{KCl}$ insulation was approximately $15 \mu \mathrm{m}$ on both sides of the sample. Two small ruby chips have also been loaded for pressure determination.

Single crystal diamond anvils often introduce significant distortions to XAS spectra due to Bragg diffraction. ${ }^{40}$ This problem is particularly important at high $\mathrm{X}$-ray energies $(\mathrm{E} \geq 8 \mathrm{KeV})$. Today nanopolycrystalline anvils are being produced ${ }^{41}$ which do not generate sharp diffraction peaks due to the small size of the crystallites. These anvils are opening the way to high pressure EXAFS studies at high energy. ${ }^{42,43}$ Due to the possibility offered by the energy-dispersive scheme to visualize the whole XAS spectrum instantly, Bragg diffraction peaks from the anvils can be moved out of the energy range of interest by changing slightly (by few degrees) the DAC orientation relative to the X-ray beam.

As shown in Fig. 6, at the Ni K-edge $(E=8.33 \mathrm{KeV})$, it is still possible to obtain high quality EXAFS spectra with $\sim 400 \mathrm{eV}$ energy range. The sample was pressurized to 64(1) GPa and then heated. In order to reduce thermal stress, mechanical instabilities, and chemical reactions of the sample (for example, carbides formation due to reaction with the diamond anvils ${ }^{44}$ ), the heating time was as short as possible. Lasers were turned on at a pre-set power for $1.2 \mathrm{~s}$, and 10 XAS spectra, each integrated for $100 \mathrm{~ms}$, were acquired and then averaged for better statistics. Simultaneously with the XAS measurements, temperature from both sides was recorded and immediately analyzed. Time synchronization between the lasers, X-ray detector, and spectrometer shutter and CCD acquisition was performed using an analog transistor-transistor logic (TTL) signal from a multichannel pulse generator. XAS and temperature measurements have been delayed by $0.1 \mathrm{~s}$ relative to the laser pulse arrival in order to achieve full thermal equilibrium in the sample. Such a strategy allows adjusting laser power in a simple and controlled manner in order to achieve uniform heating from both sides and to adjust temperature with small increments between measurements. Laser power was incremented by $0.5 \mathrm{~W}$ between temperature points.

A uniformly heated spot of approximately $15-20 \mu \mathrm{m}$ was obtained on the sample. At $\mathrm{T}=2200 \mathrm{~K}$, temperature variations $<150 \mathrm{~K}$ were measured across this area. The power of the two lasers was adjusted in order to maintain the temperatures measured on the two sides of the sample equal to within $50 \mathrm{~K}$. Temperature values remained stable during each of the $1.2 \mathrm{~s}$ heating cycles, as monitored by the video camera signal.

Although the optical setup and the CCD camera sensitivity allow temperature measurements starting from $\sim 1000 \mathrm{~K}$, with a heating duration of $\sim 1 \mathrm{~s}$ only temperatures exceeding $\sim 1600 \mathrm{~K}$ can be measured optically. This limit critically depends on the sample emissivity as well. However, when the sample remains within a single phase stability field, temperatures in the lower range can be calculated from the EXAFS spectra themselves, using the mean square relative displacement (msrd) factors obtained from the fits, as described below.

The left panel of Fig. 6 shows the obtained XAS spectra at various temperatures, normalized and shifted vertically for clarity. Upon temperature increase, the amplitudes of EXAFS oscillations decrease due to the thermal vibrations of atoms. Besides this damping effect, no changes are observed up to 2900 K. At higher temperatures, however, XAS spectra exhibit significant modifications, especially in the near-edge region, clearly indicating a phase transition. These changes are best seen in the XANES part of the spectra (Fig. 6, right), where all the experimental spectra are superimposed. The two spectra at highest temperatures (shown in red) differ from the rest: the rising edge part abruptly smooths out (arrow 1), and the doublepeak structure, typical for fcc-structured metals (arrow 2), disappears.

The observed melting point is higher than previous measurements at the same pressure (Fig. 7): 2460 K (Errandonea et al. ${ }^{37}$ ), $\sim 2490 \mathrm{~K}$ (Japel et al. ${ }^{36}$ ), or $\sim 2560 \mathrm{~K}$ (Lazor et al. ${ }^{35}$ ). However, it agrees with the most recent study (Lord et al. ${ }^{38}$ ), where $\mathrm{T}_{\mathrm{m}} \sim 3180 \mathrm{~K}$ for this pressure. Among these studies, only the latter used X-ray diffraction as a bulk probe for melting determination. The extended XAS results on the high-pressure
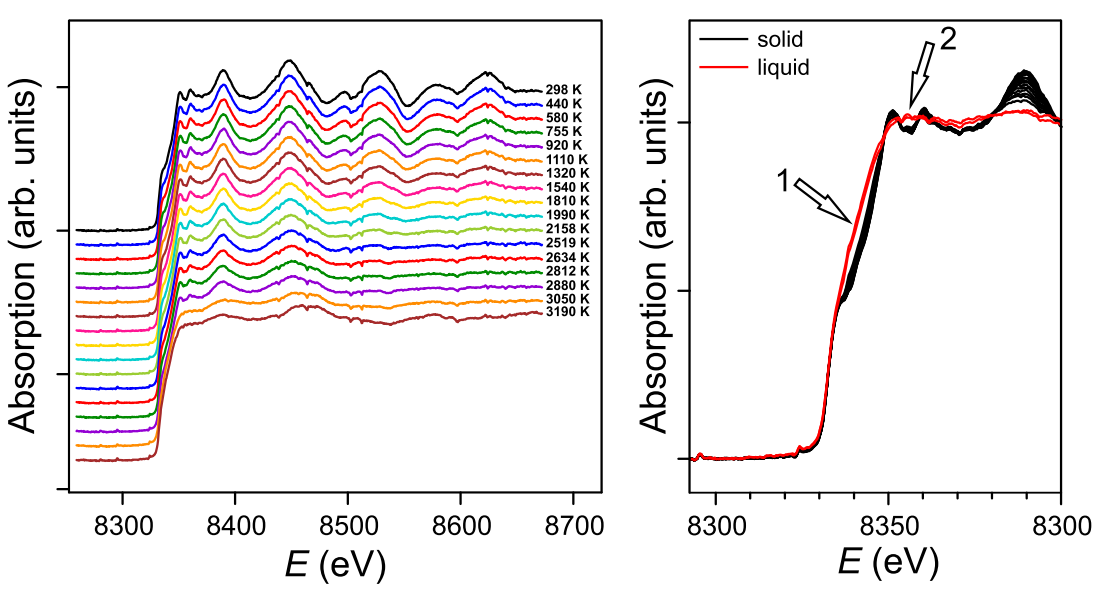

FIG. 6. Left: Normalized XAS spectra of Ni taken at $64 \mathrm{GPa}$ and temperatures from 298 to $4190 \mathrm{~K}$. Spectra are shifted vertically for clarity. Right: XANES part of the spectra, showing discontinuous changes between the solid (black) and liquid (red) phases. Two arrows show the parts of the spectra where melting is the most evident. 


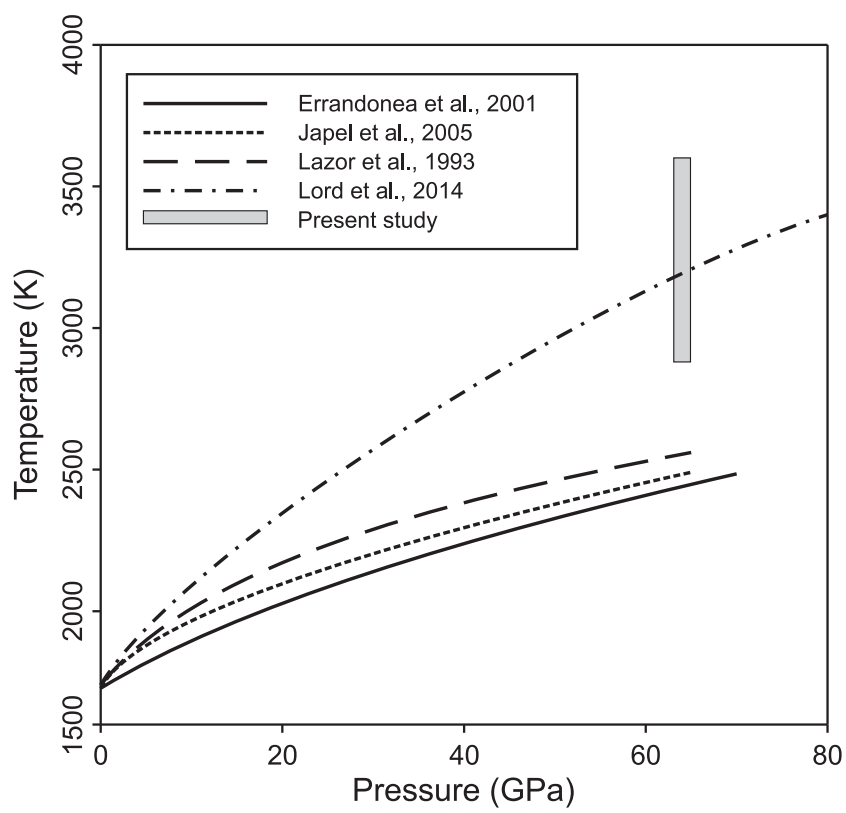

FIG. 7. Previously reported melting curves of nickel ${ }^{35-38}$ along with the results from the current study. The grey rectangle shows the temperature interval between the highest recorded temperature of solid $\mathrm{Ni}(3600 \mathrm{~K})$ and the lowest temperature of liquid $\mathrm{Ni}(2880 \mathrm{~K})$.

$\mathrm{Ni}$ melting with the present laser heating setup are given by Boccato et al. ${ }^{45}$

The EXAFS signal $\chi(k)$ from the nearest neighbors in a crystalline solid with a well-defined first coordination shell (such as $f c c \mathrm{Ni}$ ) can be expressed as

$$
\begin{aligned}
\chi(k)= & \frac{S_{0}^{2}}{k} N\left|f_{s}(k, \pi)\right| \frac{e^{-2 C_{1} / \lambda}}{C_{1}^{2}} e^{-2 k^{2} C_{2}} \\
& \times \sin \left[2 k C_{1}-\frac{4 k^{3} C_{3}}{3}+\varphi(k)\right],
\end{aligned}
$$

where $N$ is the coordination number ( $N=12$ for the $f c c$ crystal structure), $f_{s}(k, \pi)$ and $\varphi(k)$ are the atomic back-scattering amplitude and phase shift, $S_{0}^{2}$ is the amplitude reduction term (typically amounts $0.7 \div 0.9$ ), $\lambda$ is the photoelectron mean free path, and $C_{i}$ are the cumulants of the effective atomic distribution $P(r, \lambda) .{ }^{46}$

Atomic back-scattering amplitudes and phase shifts were calculated $a b$ initio using the FEFF6 software, ${ }^{47}$ while conventional data reduction and fitting have been performed using the IFEFFIT software package. ${ }^{48}$

As a result of the EXAFS fitting procedure, the three cumulants $C_{i}$ of the first coordination shell have been extracted for each temperature, while $S_{0}^{2}$ and $N$ were fixed at 0.9 and 12, respectively. The first cumulant $C_{1}$ corresponds to the mean interatomic distance $\langle r\rangle$, while $C_{2}$ and $C_{3}$ are the mean square and mean cubic relative atomic displacement, $C_{2}=$ $\left\langle(r-\langle r\rangle)^{2}\right\rangle$ and $C_{3}=\left\langle(r-\langle r\rangle)^{3}\right\rangle$.

The obtained mean square relative displacements of the atoms (also referred to as the EXAFS Debye-Waller parameter) are increasing significantly with temperature due to thermal vibrations. The temperature dependence of $C_{2}$ can be described within the framework of the Einstein model as

$$
C_{2}=\hbar^{2} \operatorname{coth}\left(\Theta_{\mathrm{E}} / 2 T\right) /\left(2 \mu k_{B} \Theta_{\mathrm{E}}\right)+C_{2(\text { static })},
$$

where $h$ and $k_{B}$ are the Planck and Boltzmann constants, $\Theta_{\mathrm{E}}$ is the Einstein temperature, $\mu$ is the reduced mass, and $C_{2 \text { (static) }}$ is the static contribution to the atomic disorder. The left panel of Fig. 8 shows the temperature variation of the $C_{2}$ and $C_{3}$ cumulants, and the red solid line represents the Einstein model fit with the assumption that $\Theta_{\mathrm{E}}$ is temperature independent. The obtained value of $\Theta_{\mathrm{E}}$ is $546 \pm 8 \mathrm{~K}$, which is fairly close to the $a b$ initio Debye temperature calculations for $\mathrm{Ni},{ }^{49}$ which varies between approximately 564 and $496 \mathrm{~K}$ in the $300-1500 \mathrm{~K}$ temperature range for the corresponding bulk density.

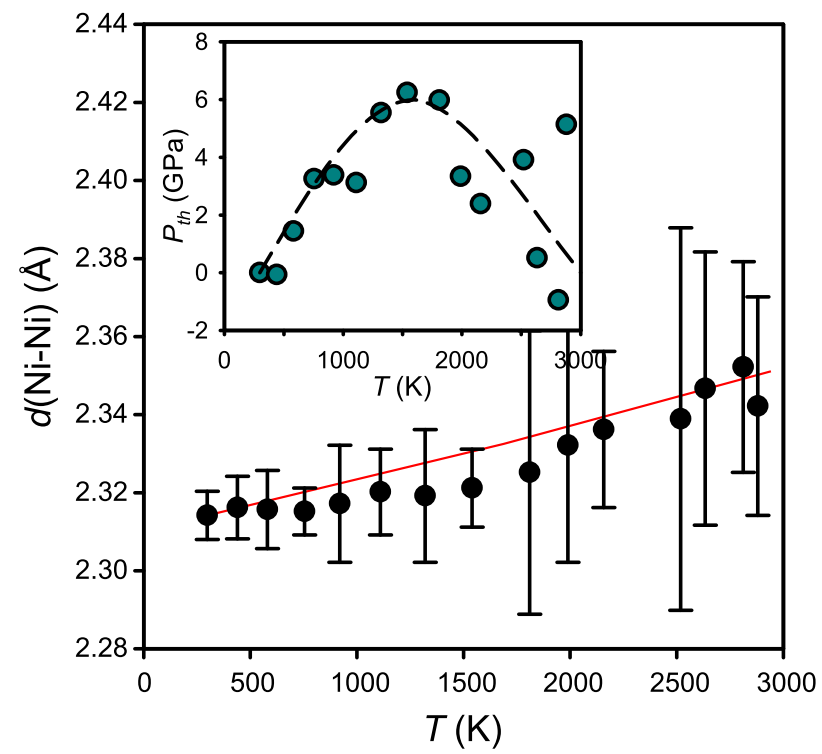

FIG. 8. Left panel: $C_{2}$ (circles) and $C_{3}$ (triangles) EXAFS cumulants for Ni at $64 \mathrm{GPa}$ as a function of temperature. The solid red line is the Einstein fit for the mean square relative displacements. Filled circles represent the $C_{2}$ values with known temperature (used for the fit), while open circles represent spectra for which temperature was not measured directly, but instead calculated from the model. Right panel: mean Ni-Ni distances at 64 GPa as a function of pressure. The solid red line shows the thermal expansion value from the Ni thermal equation of state. ${ }^{49}$ The inset shows estimated thermal pressure values. 
Although the present optical setup allows measuring temperatures below $1000 \mathrm{~K}$ (see Fig. 5) due to the short (1-2 s) exposure time used in this example, it was not possible to measure reliably temperatures below $\sim 1600 \mathrm{~K}$. Thus, for the
Einstein model fit described above, only points at ambient temperatures and $T>1600 \mathrm{~K}$ were used (black circles in Fig. 8, left panel), while the intermediate temperatures were calculated from the $C_{2}$ values and the model obtained.
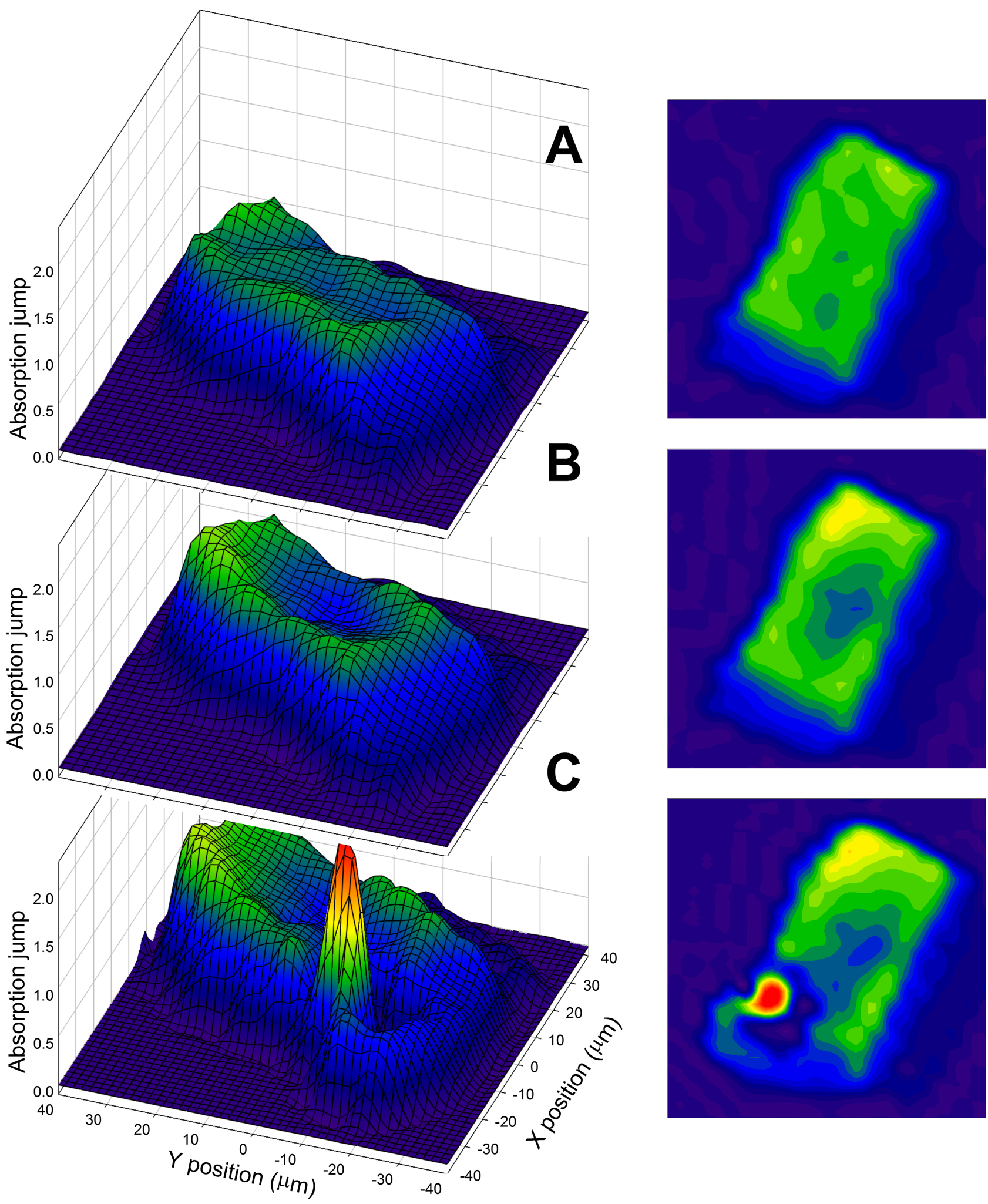

FIG. 9. Absorption jump maps of a metal foil in the diamond anvil cell before (a) and after two melting events using laser heating (b) and (c) at $\sim 30$ GPa. Left and right panels: 3D and 2D visualization of the same data set. 
For a harmonic oscillator, the mean cubic displacement is equal to zero. Thus, the absolute value of the EXAFS $C_{3}$ cumulant can be used to characterize the unharmonicity of the solid or the asymmetry of the effective interatomic potential. Triangles in the left panel of Fig. 8 show the obtained values of $C_{3}$ in $\mathrm{Ni}$ at $64 \mathrm{GPa}$ as a function of temperature. It is clear that below $\sim 1500 \mathrm{~K}$ the nickel metal can be considered as a harmonic solid, while at higher temperatures, this contribution becomes significant.

The Ni-Ni interatomic distances, obtained from the EXAFS fit, are shown in Fig. 8 (right panel). Due to the limited energy range of the measured spectra and the significantly reduced amplitude of the EXAFS oscillations at high temperatures, the uncertainty in the absolute values of $d(\mathrm{Ni}-\mathrm{Ni})$ is quite high. However, an estimate of the pressure change in the sample due to temperature increase (the thermal pressure) can be evaluated from the thermal equation of state $V=f(P, T)$ of $\mathrm{Ni}$, available in the literature ${ }^{49}$ see Fig. 8 , right panel (inset). If the sample volume is small compared to the pressure transmitting medium, and the latter is very soft, allowing the sample to expand freely, the thermal pressure is zero. The sample volume and corresponding $\mathrm{Ni}-\mathrm{Ni}$ distances then increase according to its thermal expansion (red line in Fig. 8, right panel). At the other extreme, if the pressure medium is hard and incompressible, conditions are purely isochoric (i.e., the sample volume is constant), and the internal pressure increases according to the sample thermal equation of state to compensate its inability to expand.

At low temperature (below $\sim 1700 \mathrm{~K}$ ), the volume of $\mathrm{Ni}$ remains relatively constant because the relatively hard pressure medium $(\mathrm{KCl})$ does not allow it to expand, leading to an increase of thermal pressure up to $\sim 6 \mathrm{GPa}$. At higher temperatures, however, nickel starts to expand, and thermal pressure falls down to virtually zero when approaching melting. Our estimations of thermal pressure are also comparable to the other studies. Thus, Lord et al. ${ }^{38}$ estimated the thermal pressure of $\mathrm{Ni}$ to be between $\sim 3 \mathrm{GPa}$ in $\mathrm{KCl}$ (pressure transmitting medium) and $\sim 12 \mathrm{GPa}$ in $\mathrm{MgO}$ at the melting point near $60 \mathrm{GPa}$, while the estimate of Lazor et al. ${ }^{35}$ in $\mathrm{MgO}$ was 2.7 GPa near $60 \mathrm{GPa}$.

Melting of a thin metal foil can easily modify sample geometry. Energy-dispersive XAS measurements require a flat sample with uniform thickness. As soon as the sample melts, it has a tendency to form a spherical droplet, to minimize its surface energy. Depending on the duration of melting, rigidity, and plastic deformation of the pressure medium, the molten sample can easily change its shape. The ability of the ID24 beamline to perform fast hyperspectral two-dimensional maps (where each map pixel contains a full XAS spectrum) allows easy graphic evaluation of the sample homogeneity and state. Figure 9 shows examples of the absorption jump (proportional to the sample thickness) variation before laser heating (a) and after two heating cycles (b) and (c) at $\sim 30 \mathrm{GPa}$. For the first heating cycle, the laser beam was aligned on the central part of the sample, and its geometry remained almost intact after melting. For the second cycle, the laser beam was moved towards the lower corner of the sample, and the sample shape changed drastically after melting to form a small sphere of metal and leaving a hole in a previously uniform foil.

\section{CONCLUSIONS}

We present a new facility dedicated to X-ray absorption studies of matter at extreme pressures $(\mathrm{P}>100 \mathrm{GPa})$ and temperatures $(\mathrm{T}>3000 \mathrm{~K})$ featuring double-sided laser heating of samples compressed in the diamond anvil cell. The optical scheme is designed to fully exploit the small and stable $\mathrm{X}$-ray focal spot, the energy stability, and the acquisition speed of energy dispersive XAS.

The facility is available for user operation on the energy dispersive XAS beamline of the ESRF and offers opportunities in diverse science fields, from studies of chemistry of mantle minerals at the relevant pressures and temperatures, to in situ investigation of the electronic and local structure in new materials synthesized high $P, T$ conditions.

\section{ACKNOWLEDGMENTS}

The authors would like to acknowledge Dr. Vitaly Prakapenka and Reinhard Boehler for their useful suggestions and comments on the construction and commissioning of the presented setup.

${ }^{1}$ R. Ingalls, E. D. Crozier, J. E. Whitmore, A. J. Seary, and J. M. Tranquada, J. Appl. Phys. 51, 3158 (1980).

${ }^{2}$ E. Schultz, M. Mezouar, W. Crichton, S. Bauchau, G. Blattman, D. Andrault, G. Fiquet, R. Boehler, N. Rambert, B. Sitaud, and P. Loubeyre, High Pressure Res. 25, 71 (2005).

${ }^{3}$ G. Shen, M. L. Rivers, Y. Wang, and S. R. Sutton, Rev. Sci. Instrum. 72, $1273(2001)$

${ }^{4}$ J. Zhao, W. Sturhahn, J. Lin, G. Shen, E. E. Alp, and H. Mao, High Pressure Res. 24, 447 (2004).

${ }^{5}$ Y. Meng, R. Hrubiak, E. Rod, R. Boehler, and G. Shen, Rev. Sci. Instrum. 86, 72201 (2015).

${ }^{6}$ T. Watanuki, O. Shimomura, T. Yagi, T. Kondo, and M. Isshiki, Rev. Sci. Instrum. 72, 1289 (2001).

${ }^{7}$ S. Pascarelli, O. Mathon, T. Mairs, I. Kantor, G. Agostini, C. Strohm, S. Pasternak, F. Perrin, P. Chapellet, and C. Clavel, J. Synchrotron Radiat. 23, 353 (2016).

${ }^{8}$ R. Ingalls, G. A. Garcia, and E. A. Stern, Phys. Rev. Lett. 40, 334 (1978).

${ }^{9}$ A. Dewaele, M. Mezouar, N. Guignot, and P. Loubeyre, Phys. Rev. B 76, 144106 (2007).

${ }^{10}$ V. B. Prakapenka, A. Kubo, A. Kuznetsov, A. Laskin, O. Shkurikhin, P. Dera, M. L. Rivers, and S. R. Sutton, High Pressure Res. 28, 225 (2008).

${ }^{11}$ B. Kiefer and T. S. Duffy, J. Appl. Phys. 97, 114902 (2005).

${ }^{12}$ S. Pascarelli and O. Mathon, Phys. Chem. Chem. Phys. 12, 5535 (2010).

${ }^{13}$ J. P. Itie, A. Polian, G. Calas, J. Petiau, A. Fontaine, and H. Tolentino, Phys. Rev. Lett. 63, 398 (1989).

${ }^{14}$ A. Polian, J. P. Itie, E. Dartyge, A. Fontaine, and G. Tourillon, Phys. Rev. B 39, 3369 (1989).

${ }^{15}$ H. Tolentino, F. Baudelet, E. Dartyge, A. Fontaine, A. Lena, and G. Tourillo, Nucl. Instrum. Methods Phys. Res., Sect. A 289, 307 (1990).

${ }^{16}$ J. Pellicer-Porres, A. San Miguel, and A. Fontaine, J. Synchrotron Radiat. 5, 1250 (1998).

${ }^{17}$ S. Pascarelli, O. Mathon, and G. Aquilanti, J. Alloys Compd. 362, 33 (2004).

${ }^{18}$ A. San Miguel, H. Libotte, J. P. Gaspard, M. Gauthier, J. P. Itié, and A. Polian, Eur. Phys. J. B 17, 227 (2000).

${ }^{19}$ S. Pascarelli, G. Aquilanti, W. A. Crichton, T. Le Bihan, M. Mezouar, S. De Panfilis, J. P. Itié, and A. Polian, Europhys. Lett. 61, 554 (2003).

${ }^{20}$ G. Aquilanti and S. Pascarelli, J. Phys.: Condens. Matter 17, 1811 (2005).

${ }^{21}$ A. San-Miguel, H. Libotte, M. Gauthier, G. Aquilanti, S. Pascarelli, and J.-P. Gaspard, Phys. Rev. Lett. 99, 15501 (2007).

${ }^{22}$ D. Andrault, M. Muñoz, N. Bolfan-Casanova, N. Guignot, J.-P. Perrillat, G. Aquilanti, and S. Pascarelli, Earth Planet. Sci. Lett. 293, 90 (2010).

${ }^{23}$ R. Boehler, H. G. Musshoff, R. Ditz, G. Aquilanti, and A. Trapananti, Rev. Sci. Instrum. 80, 45103 (2009).

${ }^{24}$ L. Dubrovinsky, K. Glazyrin, C. McCammon, O. Narygina, E. Greenberg, S. Ubelhack, A. I. Chumakov, S. Pascarelli, V. Prakapenka, J. Bock, and N. Dubrovinskaia, J. Synchrotron Radiat. 16, 737 (2009). 
${ }^{25}$ G. Aquilanti, A. Trapananti, A. Karandikar, I. Kantor, C. Marini, O. Mathon, S. Pascarelli, and R. Boehler, Proc. Natl. Acad. Sci. U. S. A. 112, 12042 (2015).

${ }^{26}$ A. Salamat, R. A. Fischer, R. Briggs, M. I. McMahon, and S. Petitgirard, Coord. Chem. Rev. 277-278, 15 (2014).

${ }^{27}$ M. J. Walter and K. T. Koga, Phys. Earth Planet. Inter. 143-144, 541 (2004).

${ }^{28} \mathrm{M}$. Born and E. Wolf, Principles of Optics: Electromagnetic Theory of Propagation, Interference, and Diffraction of Light (Cambridge University Press, Cambridge, 1999).

${ }^{29}$ R. J. Hemley, H.-K. Mao, G. Shen, J. Badro, P. Gillet, M. Hanfland, and D. Hausermann, Science 276, 1242 (1997).

${ }^{30}$ R. J. Hemley, H. Mao, and S. Merkel, Appl. Phys. Lett. 74, 656 (1999).

${ }^{31}$ O. Hignette, private communication (2017).

${ }^{32}$ A. Dewaele, G. Fiquet, and P. Gillet, Rev. Sci. Instrum. 69, 2421 (1998).

${ }^{33}$ R. Torchio, Y. O. Kvashnin, S. Pascarelli, O. Mathon, C. Marini, L. Genovese, P. Bruno, G. Garbarino, A. Dewaele, F. Occelli, and P. Loubeyre, Phys. Rev. Lett. 107, 237202 (2011).

${ }^{34}$ I. Sergueev, L. Dubrovinsky, M. Ekholm, O. Y. Vekilova, A. I. Chumakov, M. Zając, V. Potapkin, I. Kantor, S. Bornemann, H. Ebert, S. I. Simak, I. A. Abrikosov, and R. Rüffer, Phys. Rev. Lett. 111, 157601 (2013).

${ }^{35}$ P. Lazor, G. Shen, and S. K. Saxena, Phys. Chem. Miner. 20, 86 (1993).

${ }^{36}$ S. Japel, B. Schwager, R. Boehler, and M. Ross, Phys. Rev. Lett. 95, 167801 (2005).

${ }^{37}$ D. Errandonea, B. Schwager, R. Ditz, C. Gessmann, R. Boehler, and M. Ross, Phys. Rev. B 63, 132104 (2001).
${ }^{38}$ O. T. Lord, I. G. Wood, D. P. Dobson, L. Vočadlo, W. Wang, A. R. Thomson, E. T. H. Wann, G. Morard, M. Mezouar, and M. J. Walter, Earth Planet. Sci. Lett. 408, 226 (2014).

${ }^{39}$ D. Zhang, J. M. Jackson, J. Zhao, W. Sturhahn, E. E. Alp, T. S. Toellner, and M. Y. Hu, Rev. Sci. Instrum. 86, 13105 (2015).

${ }^{40}$ O. Shimomura, T. Fukamachi, T. Kawamura, S. Hosoya, S. Hunter, and A. Bienenstock, Jpn. J. Appl. Phys., Part 1 17, 221 (1978).

${ }^{41}$ T. Irifune, A. Kurio, S. Sakamoto, T. Inoue, and H. Sumiya, Nature 421, 599 (2003).

${ }^{42}$ S. De Panfilis, F. Gorelli, M. Santoro, L. Ulivi, E. Gregoryanz, T. Irifune, T. Shinmei, I. Kantor, O. Mathon, and S. Pascarelli, J. Chem. Phys. 142, 214503 (2015).

${ }^{43}$ A. Dewaele, N. Worth, C. J. Pickard, R. J. Needs, S. Pascarelli, O. Mathon, M. Mezouar, and T. Irifune, Nat. Chem. 8, 784 (2016).

${ }^{44}$ V. Prakapenka, G. Shen, and L. Dubrovinsky, High Temp. - High Pressures 35/36, 237 (2003).

${ }^{45}$ S. Boccato, R. Torchio, I. Kantor, G. Morard, S. Anzellini, R. Giampaoli, R. Briggs, A. Smareglia, T. Irifune, and S. Pascarelli, "The melting curve of nickel up to 100 GPa explored by XAS," J. Geophys. Res. Solid Earth (published online).

${ }^{46}$ P. Fornasini, F. Monti, and A. Sanson, J. Synchrotron Radiat. 8, 1214 (2001).

${ }^{47}$ S. I. Zabinsky, J. J. Rehr, A. Ankudinov, R. C. Albers, and M. J. Eller, Phys. Rev. B 52, 2995 (1995).

${ }^{48}$ B. Ravel and M. Newville, J. Synchrotron Radiat. 12, 537 (2005).

${ }^{49}$ Z.-Y. Zeng, C.-E. Hu, L.-C. Cai, and F.-Q. Jing, Phys. B 407, 330 (2012). 